\title{
Propuesta de una herramienta de emprendimiento en el diseño de un modelo de negocio
}

\section{Proposal for an Entrepreneur Tool in the Design of a Business Model}

Luis-Alejandro Gazca-Herrera'; José-Rafael Gómez-Cabañas²; Jessica Garizurieta-Bernabe ${ }^{3}$; Fredy Castro-Naranjo ${ }^{4}$

\section{Palabras claves:}

Modelo de negocios, Modelo Canvas, Design

Thinking, Lean Startup

Artículo de investigación:

Fecha de recepción:

2019/11/28

Fecha de aceptación:

2019/12/20

Esta publicación se encuentra bajo licencia:

Creative Commons ReconocimientoNoComercialSinObraDerivada 4.0 Internacional

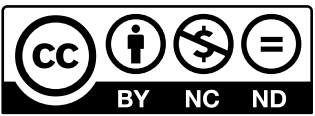

\section{Resumen}

La evolución del emprendimiento ha llevado al desarrollo y aplicación de diversas herramientas que permiten el logro de sus metas. Actualmente, los emprendedores consideran a la innovación y al modelo de negocios como dos objetivos fundamentales de su estructura por lo que hacen uso de metodologías que les permiten crear estrategias y propuestas de valor que generan un impacto positivo en la aceptación de sus productos y servicios. Design thinking, Lean startup y Business model canvas, representan tres instrumentos revolucionarios para el diseño e innovación de empresas y productos. Su uso ha sido popularizado por grandes empresas como Apple o IDEO. La presente investigación hace una revisión bibliográfica acerca de las tres herramientas de emprendimiento más utilizadas en México: Canvas, Design Thinking y Lean Startup; con el objetivo de analizar su estructura de aplicación y proponer un modelo de 11 pasos que

1 Licenciado en Sistemas Computacionales Administrativos, Maestro en Ciencias Administrativas y Doctor en Administración Pública, Universidad Veracruzana, México, @Alex_Gazca, Igazca@uv.mx,código ORCID: https:// orcid.org/0000-0001-7637-2909

2 Licenciado en Administración, Maestría en Gestión de las Organizaciones, Universidad Veracruzana, México, rgomez@uv.mx, código ORCID: https:// orcid.org/0000-0002-2411-9669

3 Licenciada en Adeministración, Maestra en Finanzas y Doctora en Desarrollo Económico y Sectorial Estratégico, Universidad Veracruzana, México, @ jessgarizurieta, jgarizurieta@uv.mx, código ORCID: http://orcid.org/00000002-1443-4737

4 Licenciado en Contaduría y Doctor en Administración Empresarial, Universidad Veracruzana, México, @fredycn, fcastro@uv.mx, 


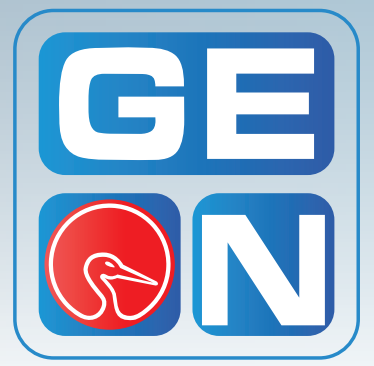

\section{Revista GEON}

(Gestión, Organizaciones y Negocios)

ISSN: 2346-3910 en línea

revistageon@unillanos.edu.co

Universidad de los Llanos

Colombia

Gazca Herrera, L., Gómez Cabañas, J., Garizurieta Bernabe, J., \& Castro Naranjo, F. (2020).

Propuesta de una herramienta de emprendimiento en el diseño de un modelo de negocio

Revista GEON

(Gestión, Organizaciones y Negocios), 7(1), 96-111.

\section{토요}

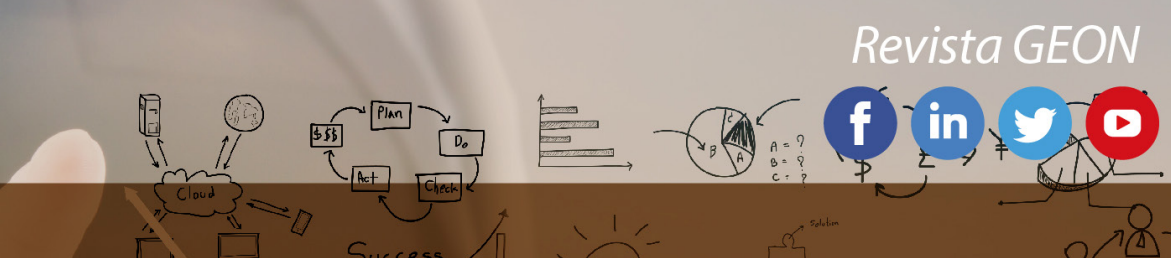

66 la propuesta del Modelo de Herramienta de Emprendimiento, permite aplicar a través de un solo modelo tres métodos, fortaleciendo el proceso previo al emprendimiento

Gažca Herrera, L, Gómez Cabañas, 90: (Garizurieta Bernabe, I., \& Castro Naranjo, F. (2020) I. ropuesta de una herramienta de emprendimiento en el diseño de un modelo de negocio. Revista GEON (Gestión, Organizaciones Y Negocios), 7(1), 96-111.
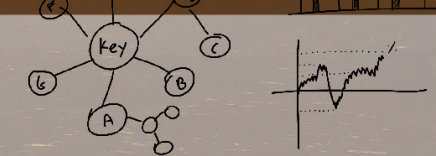

http://revistageon.unillanos.edu.co 
permita medir el impacto que tienen las soluciones basadas en el diseño de un modelo de negocio, de una manera más integral.

Palabras claves: Modelo de negocios, Modelo Canvas, Design Thinking, Lean Startup

\section{Abstract}

The evolution of the entrepreneurship has led to the development and application of different tools that allow the achievement of their goals. Currently, the entrepreneurs consider innovation and business models as two fundamental objectives of their structure, so they make use of methodologies that allow them to create strategies and value propositions that generate a positive impact on the acceptance of their products and services. Design thinking, Lean startup and Business model canvas represent three revolutionary instruments for the design and innovation of business and products. Its use has been popularized by large companies such as Apple or IDEO. The present investigation makes a documentary review about the three most used entrepreneurial

Cómo citar este artículo / To reference this article:

Gazca Herrera, L., Gómez Cabañas, J., Garizurieta Bernabe, J., \& Castro Naranjo, F. (2020). Propuesta de una herramienta de emprendimiento en el diseño de un modelo de negocio. Revista GEON (Gestión, Organizaciones Y Negocios), 7(1), 96-111. https://doi. org/10.22579/23463910.200 tools in Mexico: Canvas, Design Thinking and Lean Startup; in order to analyze its application structure and propose an 11-step model that allows measuring the impact of solutions based on the design of a business model, in a more comprehensive way.

Keywords: Bussines Model Canvas, Design Thinking, Lean Startup, Business Model.

\section{Introducción}

De acuerdo con Cervilla y Puente (2013) citando a Hisrich (2006), el emprendimiento "es el proceso llevado a cabo por un individuo para crear algo que agregue valor, asignando el tiempo y el esfuerzo necesario, asumiendo los riesgos financieros, sociales y sicológicos asociados, y recibiendo la recompensa resultante en términos monetarios, de satisfacción personal y de indepencia" (p:10).

En el mismo sentido Cervilla y Puente (2013) citando a el Global Entrepre- 
neurship Monitor (GEM) (Bosma et al., 2008), se define al emprendedor como una persona capaz de percibir una oportunidad y asumir los riesgos existentes para crear nuevos mercados, diseñar nuevos productos y desarrollar procesos innovadores. Continua mencionando que este estudio internacional adopta una visión amplia del emprendimiento y se enfoca en el papel desempeñado por los individuos en el proceso emprendedor, distinguiendo entre quienes emprenden por oportunidad, es decir, deciden iniciar una actividad empresarial cuando identifican una oportunidad de negocio luego de una búsqueda sistemática, y los que emprenden por necesidad porque no tienen otra alternativa, observándose que en prácticamente todos los países estudiados en el GEM la motivación principal para emprender se da por oportunidad (IESA, 2008).

El emprendimiento en la actualidad representa un área de oportunidad para todos aquellos que deseen emprender un negocio, sin embargo, es necesaria la aplicación de herramientas o modelos que les permitan alcanzar sus objetivos y que las empresas que se generen a partir de dichas intenciones tengan una vida de desarrollo a largo plazo.

Se reitera la importancia que el emprendimiento debe ir acompañado de estrategias que permitan crear negocios con una sobrevivencia larga, ya que de acuerdo con Pérez, Cruz, Guatemala, \& Juárez (2018) citando Perina (2018), de conformidad con los datos estandarizados muestra que durante el primer año de arranque mueren el 50\% de las pequeñas y medianas empresas; para el segundo año suman otro $25 \%$ y para el quinto año muere otro 15\%, quedando sólo un $10 \%$ del total que sobrevivirá madurando como pequeña empresa; lo anterior como resultado probablemente por una toma de decisiones de forma empírica o bien a la falta del uso de una herramienta de emprendimiento en su diseño de modelo de negocio inicial, en el mismo sentido Torralba , Pérez , \& Martínez (2017) establecen que al iniciar un nuevo proyecto de emprendimiento la quiebra empresarial (fracaso)es uno de los problemas que más preocupan a los nuevos empresarios; ya que se deben realizar muchos esfuerzos logrando pocos avances.

Con base en lo anterior, es que se llevó a cabo la siguiente investigación, la cual está conformada en dos apartados; en el primero se realiza la revisión documental respecto a las tres herramientas de emprendimiento utilizadas comúnmente por los mexicanos: design thinking, lean startup and Business Model CANVAS.

Posteriormente, en un segundo apartado, se realiza un análisis de la estructura de cada una de estas herramientas, con la finalidad de desarrollar una propuesta de modelo de aplicación de estas, para ofrecer soluciones basadas en el diseño de un modelo de negocio, de una manera más integral.

\section{Desarrollo}

De acuerdo con Kundel (1991) Citado en Formichella (2004), "la actividad 
emprendedora es la gestión del cambio radical y discontinuo, o renovación estratégica, sin importar si esta renovación estratégica ocurre adentro o afuera de organizaciones existentes, y sin importar si esta renovación da lugar, o no, a la creación de una nueva entidad de negocio".

Ahora bien, en cuanto a los modelos de negocios Demil \& Lecocq (2009) mencionan que el término "modelos de negocio" ha prosperado en la bibliografía dedicada a las actividades gerenciales desde finales de la década de 1990, especialmente a raíz de la aparición de la era de Internet y de su adopción masiva por parte del comercio (Ghaziani y Ventresca, 2005). Continúan mencionando que, en los discursos de los directivos, los modelos de negocio se suelen mencionar para evocar la idea de cambio, en frases como esta: "Tenemos que hacer evolucionar nuestro modelo de negocio" (véase, por ejemplo, Yip, 2004 o Johnson y cols., 2008). Un modelo de negocio, raras veces se descubre de inmediato, para crear una coherencia interna o adaptarse al entorno, por lo que, es necesario realizar sucesivos ajustes. Demil \& Lecocq citando a Winter y Szulanski (2001: 731) argumentan: "La fórmula o el modelo de negocio, lejos de ser una cantidad de información que se revela de una sola vez, normalmente es un conjunto complejo de rutinas interdependientes que se descubren, ajustan y matizan mediante la acción". Algunos cambios masivos en el modelo de negocio pueden incluso transformar radicalmente un sector industrial. Por ejemplo, la aparición de un sector de prensa gratuita se describe a menu- do como uno de los factores del declive de los medios de comunicación tradicionales.

A continuación, se define cada una de las herramientas anteriormente mencionadas, junto con su modelo de aplicación.

\section{Design thinking}

Design Thinking es un método analítico y creativo (Razzouk, 2012) tanto para resolver problemas complejos como para la innovación centrada en el usuario, por medio de un equipo multidisciplinario (Thoring, 2012), el concepto se inspira en la forma de trabajo de los diseñadores cuya aplicación fue trasladada hacia la administración (Carlgren \& Elmquist, 2016), su popularización en el ámbito de los negocios y el emprendimiento se debe a David Kelley; fundador de la compañía IDEO, Tim Brown; CEO y presidente de IDEO y a Roger Martin, ex decano de Rotman School (Brown, 2008).

Desde una perspectiva analítica la investigación de Design thinking puede ser clasificada en dos formas (WoodiIla \& Çetinkaya, 2013):

1. 'Designerly thinking'. En este ámbito se hace referencia a la perspectiva profesional de los diseñadores, estudios sobre la competencia que tienen en la teoría, la práctica y las reflexiones sobre como caracterizar e interpretar dicha habilidad.

2. 'Design thinking'. En este ámbito se hace referencia a personas relacionadas en particular con áreas de la 
administración, es decir, cuando se aplica en otras áreas por personas que no tienen el mismo trasfondo de estudios en diseño.

La rama que se pretende profundizar es aquella del 'Design thinking'. Este método puede ser estudiado en diversos enfoques debido a su amplia gama de investigaciones en diversas áreas del conocimiento. En primer aspecto se analiza su forma más sencilla como un sistema basado en tres etapas propuestas por Tim Brown (2009):

1. Inspiración: Aquella situación por la que se busca una solución.

2. Ideación: Proceso de crear y probar ideas.

3. Implementación: Fase que hace evolucionar desde la etapa de ideas hasta su implementación en el mercado.

Otro enfoque es aquel elaborado en la rama de la ingeniería (MÜLLER, 2011), siendo un modelo más desarrollado basado en seis etapas:

1. Entender: Se refiere a la etapa de recolección y análisis de la información existente sobre el área que se pretende analizar.

2. Observación: Recopila y analiza toda la información sobre las necesidades de los usuarios por métodos de búsqueda cuantitativos que permitan definir el problema.

3. Punto de vista: Crea una teoría sobre las necesidades de los usuarios abordado desde todas las perspectivas posibles.
4. Ideación: Crea posibles soluciones a dicho problema por medio de técnicas que permitan crear una gama amplia de opciones.

5. Prototipo: Selecciona diversas opciones y las desarrolla a un mayor nivel, creando representaciones que permitirán materializar dicha idea.

6. Prueba: Fase que permite mostrar el prototipo, probar y analizar su comportamiento ante sus usuarios para así poder obtener retroalimentación de las partes interesadas.

Una tercera perspectiva es propuesta por Stanford (2010) compuesta por cinco etapas:

1. Empatizar: Se considera como el fundamento del proceso de diseño centrado en el usuario a través de tres conceptos clave, la observación, el compromiso y la inmersión, el motivo de esta etapa es la de entender a aquellos para los que diseñas; ya que generalmente los que diseñan una solución para los problemas generalmente no son los mismos que tienen los problemas, por lo que se debe construir empatía para comprender el contexto y la importancia de su solución.

2. Definir: Una vez lograda la empatía con la gente para la que diseñas, se definen las características de aquellas necesidades de dicha población para así identificar desde tu punto de vista aquellos aspectos importantes que debes cubrir para realmente enfocarte en tus usuarios. 
3. Idear: En esta fase se tiene el objetivo de la creación de alternativas visto desde una perspectiva holística, generar un espacio amplio de soluciones sin profundizar tanto en cada una de estas, esto es así ya que una vez creada la gamma de opciones se puede comenzar a crear prototipos a partir de ellos. Un aspecto importante es poder generar ideas con flexibilidad y variedad de cambios en los que puedes abordar la solución.

\section{Prototipo: Crear prototipos en} esta fase es llevar a la realidad las ideas, en general se propone mantenerlas básicas y rápidas de hacer para poder analizar dicha solución, investigar más sobre aquellos aspectos en particular que se requerirían para llevarse a cabo y al mismo tiempo tener la posibilidad de investigar otras alternativas.

5. Prueba: Realizar pruebas de los prototipos permite obtener retroalimentación, realizar ésta permite encontrar aquellas áreas a mejorar de dicho prototipo o la posibilidad de generar nuevas ideas con una mayor orientación hacia lo que se necesita.

En conclusión, se puede observar como esta metodología permite la resolución de problemas complejos, proporcionando en la mayoría de las veces, una respuesta creativa a las situaciones complejas que se presenten.

\section{Lean startup}

La siguiente herramienta para analizar es Lean Startup, que como es po- sible vislumbrar, es una herramienta excelente para la creación de prototipos en torno a la solución, pero proporciona menos orientación en la generación de ideas. Este enfoque que surgió con el objetivo de crear innovaciones continuas (Ries \& Julián, 2012), una metodología que ha tomado mayor uso en los últimos años y que ha sido abordada por diversos autores, tales como: Eric Ries (2012) o Ash Maurya (2012), la publicación de artículos en los cuales se describen formas de crear educación emprendedora y tácticas para poder enseñar lean startup en clase (Craig E. Armstrong, 2016), o su aplicación en diversas áreas, tales como ciencias de la salud, en temas de insuficiencia cardiaca, o en el área de administración, en la resolución de problemáticas dentro de las organizaciones. (Amber E. Johnson et al., 2014).

Basado en lean maufacturing (Thor ing, 2012) design thinking (Brown, 2008), customer development (Blank, Blank, \& Dorf, 2012) y agile development (Shore, 2007), esta técnica centrada en el usuario hace uso de ciclos de tiempo cortos, un enfoque científico para la toma de decisiones y en general una metodología aplicable para startups o grandes empresas.

Aunque su enfoque en primera instancia es para el desarrollo de software, las características del mismo modelo; tomando como base a Ries (2012), pueden ser aplicables a cualquier ámbito, esta consta de tres pasos llamado 'circuito feedback':

1. Crear: Es considerado como el proceso de convertir ideas en productos. 
2. Medir: Realizar una medición de la respuesta de los consumidores.

3. Aprender: A perseverar o a 'pivotar' dicha idea o producto.

En general el libro escrito por Ries se divide en tres partes, mismas que consideramos importante para entender al modelo Lean Startup:

1. Ver: Donde propone el 'conocimiento validado' como parte fundamental, misma que a grandes rasgos hace alusión a la experimentación científica.

2. Dirigir: Parte donde se describe el método de Lean Startup y su aplicación a través del 'circuito feedback'.

3. Acelerar: Aplicaciones del circuito feedback con el método lean Startup.

Como fue posible observar, esta herramienta permite llegar a las soluciones de manera más ágil, acortando los pasos a seguir en el proceso, sin embargo, se piensa que no desarrolla en su totalidad la creatividad.

\section{Modelo Business Model CANVAS}

Es aquí donde entra el modelo Business Model CANVAS, el cual conlleva a una visión general de todos los componentes para entregar y capturar valor al usuario. Business Model CANVAS es una metodología nueva de modelo de negocio (Osterwalder \& Pigneur, 2011) cuya aplicación ha tomado un mayor uso hoy en día.
Con base en Ostelwader \& Pigneur se explicará dicho modelo.

Representa una forma de planificación para la creación de valor de una empresa basada en 9 aspectos, estos se agrupan en una representación gráfica, cada uno de los módulos que lo conforman representa un área clave de atención para lograr un modelo de negocio, estos son:

1. Segmentos de mercado: Aquella sección del mercado el cual se atenderá, en esta parte se define al tipo de cliente al cual va dirigido dicho producto, segmentar al mercado potencial y describir las características que dicho cliente debe tener.

2. Propuestas de valor: Aquel bien o servicio que se ofrecerá a tu segmento de mercado, incluyendo la ventaja que se ofrece al cliente sobre dicho producto o servicio que se ofrecerá a diferencia de aquellos que ya existen en el mercado.

3. Canales: La forma en que se realizará el contacto entre la empresa-cliente para hacer llegar su producto, ya sea desde darlo a conocer hasta la atención posventa, los canales representan un aspecto fundamental para hacerles Ilegar la propuesta de valor.

4. Relaciones con clientes: Es el nexo que se crea con cada segmento de mercado al que se atiende dependiendo del bien o servicio que se ofrezca, en este aspecto se puede ayudar a la fidelización o estimulación de ventas con los clientes. 
5. Fuentes de ingresos: La forma de obtención de dinero, en esta sección se describe aquella manera en que trabaja la empresa para conseguir su fuente de recursos, siendo esta la más adecuada para su producto y al mismo tiempo para el mercado al que se dirige.

6. Recursos clave: Aquellos aspectos más importantes que son necesarios para que funcione cada una de las partes de la empresa.

7. Actividades clave: Acciones necesarias a llevar a cabo para el funcionamiento del modelo de negocios.

8. Asociaciones clave: Representa las alianzas principales a tomar en cuenta que hacen posible el correcto desempeño del modelo de negocios.

9. Estructura de costos: Todos los gastos necesarios a tomar en cuenta para el funcionamiento del modelo de negocios.

Dentro del mismo esquema se puede crear un enfoque en dos módulos, al análisis de la propuesta de valor.

Compuesta por dos secciones denominadas perfil del consumidor; del inglés Customer Profile, y en mapa de valor (del inglés value map) es una manera de describir a mayor detalle la forma en que se entregará valor al cliente.

En lasección de "Value Map" cuya forma cuadrada se divide en tres secciones, representa aspectos denominados:

1. Products \& Services: Aquellos productos en los cuales se enfoca la propuesta de valor a analizar.
2. Gain Creators: Aquella sección en la que se describe como el producto crea valor para el consumidor.

3. Pain Relievers: Sección en la que se describe cómo es que el bien o servicio puede solucionar un problema del consumidor.

Dentro de la sección de "Customer Profile" se analizan tres rubros:

1. Customer Jobs: Sección donde se describen las actividades que el cliente intenta lograr en su ámbito personal y profesional, este debe estar expresado en palabras del mismo cliente.

2. Gains: Aquello que quieren lograr o determinados beneficios que buscan.

3. Pains: Aquello que impide el logro de sus objetivos en su ámbito laboral o personal.

Dicha metodología se determina "FIT" al punto en que convergen ambas secciones, donde se logran los objetivos planteados en cada una de las áreas que se analizaron.

Por otra parte, en este modelo de negocios se propone un esquema para analizar al perfil del cliente con mayor detalle, este se apoya del siguiente material:

Compuesto por seis secciones, esta herramienta pretende; por medio de estos rubros, obtener una base para analizar el perfil del consumidor desde un aspecto sociológico. Creado por XPLANE toma ámbitos de interacción de la persona con su medio am- 
biente, desde su entorno de trabajo, amigos, mercado, aspiraciones, acciones y los resultados que desea lograr.

\section{Propuesta de Herramienta de Emprendimiento en el Diseño de un Modelo de Negocios}

De acuerdo con Fino Garzón (2013), pese a que el modelo de negocio Business Model CANVAS lleva más tiempo discutiéndose y aplicándose, la adopción y expansión de los modelos Design Thinking y Lean Startup demuestra que, aunque ambos se pueden aplicar a los Startup, también es posible que se beneficien de ellos empresas nuevas y existentes.

Como se observó anteriormente, el modelo Business Model CANVAS divide el modelo de negocio en nueve componentes básicos, permitiendo que el equipo de desarrollo evalué los nueve elementos primero de manera separada y luego de forma conjunta, con la finalidad de facilitar nuevas ideas que no hubieran sido posibles sin esta perspectiva holística. Como parte de un enfoque Lean Startup, el modelo de negocio Business Model CANVAS funciona como una herramienta de convergencia para el desarrollo de la idea, al permitir validar las hipótesis del modelo de negocio hasta que encuentre una que sea repetible y escalable, garantizando que el equipo no pase por alto elementos clave tales como canales, propuestas de valor y recursos que se necesitan. Mientras que, el uso del modelo Business Model CANVAS en combinación con el modelo de Design Thinking, permite conceptualizar la idea más como un negocio que como una so- lución, proporcionando al equipo una encapsulación visual del progreso que captura las complejidades de los diferentes elementos y sus relaciones a medida que se genera la idea.

Si se analiza el proceso de desarrollo de los tres modelos, es posible observar en su estructura tres elementos clave:

1. Clientes. Ponen énfasis en la definición de los segmentos que se van a atender, con el objetivo principal de entender el mercado y las relaciones con los clientes.

2. Estrategia. En ella se establece una propuesta de valor en términos cuantitativos y cualitativos, se determina un prototipo, la infraestructura necesaria y las fuentes de financiamiento.

3. Aplicación. Consiste en la comprensión, creatividad y análisis constructivo orientado al prototipo que permite desarrollar la idea paso a paso.

Aśi bien, a través de la identificación de estos tres elementos, es posible medir cualitativamente el impacto que tienen las soluciones basadas en el diseño de un modelo de negocio. Incluso, es posible generar un marco de evaluación cualitativa y/o cuantitativa, para entender cuáles son los cambios que aporta y medirlos para desafiarlos constantemente. De esta forma, se propone un modelo de Herramienta de Emprendimiento de once pasos (ver tabla1):

1. Explorar. El primer paso consiste en determinar si realmente existe una necesidad sin cubrir dentro 
del mercado a través de conocer la sociedad en donde se encuentra inmerso el cliente potencial, con la finalidad de saber sus gustos, preferencias, posición económica, atributos, entre otros.

2. Sumergirse. Una vez que se tiene identificada la necesidad, es necesario investigar a profundidad los detalles puntuales que le permitan al emprendedor determinar las características particulares que deberá cubrir el producto o servicio, para satisfacer la necesidad existente.

3. Idear. En este paso, se deben sentar las bases que permitan desarrollar el producto o servicio desde la óptica del cliente, de tal forma que pueda apreciarse su viabilidad y funcionalidad dentro del mercado, a través del continuo contacto con el cliente.

4. Prototipo. A partir de las ideas generadas en el paso anterior, se procese a desarrollar una primera propuesta de producto o servicio de una forma viable, flexible, iterativa, incorporando nuevas funcionalidades a medida que se desenvuelve.

5. Prueba. Este paso consiste en comprobar si las ideas se fundamentan en las necesidades reales, mismas que fueron analizadas con anterioridad, medir cómo responden los consumidores y a partir de esos datos, tomar las decisiones apropiadas.

6. Elaborar. En esta etapa se deben seleccionar las ideas recolectadas en el paso anterior y a partir de es- tas, generar un segundo prototipo que represente un mayor acercamiento a la funcionalidad del producto o servicio.

7. Prueba. Una vez que se le han realizado las modificaciones pertinentes al prototipo, es necesario llevar a cabo una validación de funcionalidad en comparación con la demanda existente. Si en la prueba resulta viable la propuesta se procede al siguiente paso, en caso contrario, se debe realizar de nuevo la elaboración del prototipo atendiendo las observaciones.

8. Perseverar. Si la funcionalidad del producto o servicio cumple con la satisfacción del usuario, se hace necesario definir formalmente el producto o servicio a ofrecer y el mercado cuya demanda busca satisfacerse.

9. Actividades. Una vez que el producto o servicio se encuentra listo para la venta, se deben determinar la serie de actividades fundamentales que le darán la pauta al negocio para posicionarse en el mercado.

10. Recursos. A partir de la definición del producto o servicio, se establecen los recursos materiales, técnicos, humanos y financieros esenciales para el desarrollo y posicionamiento del producto o servicio.

11. Estructura de costos. Finalmente, con el objetivo de establecer precios competitivos, se debe llevar a cabo la detección de los costos que se involucran en el desarrollo producto o servicio y que hacen posible su posicionamiento. 


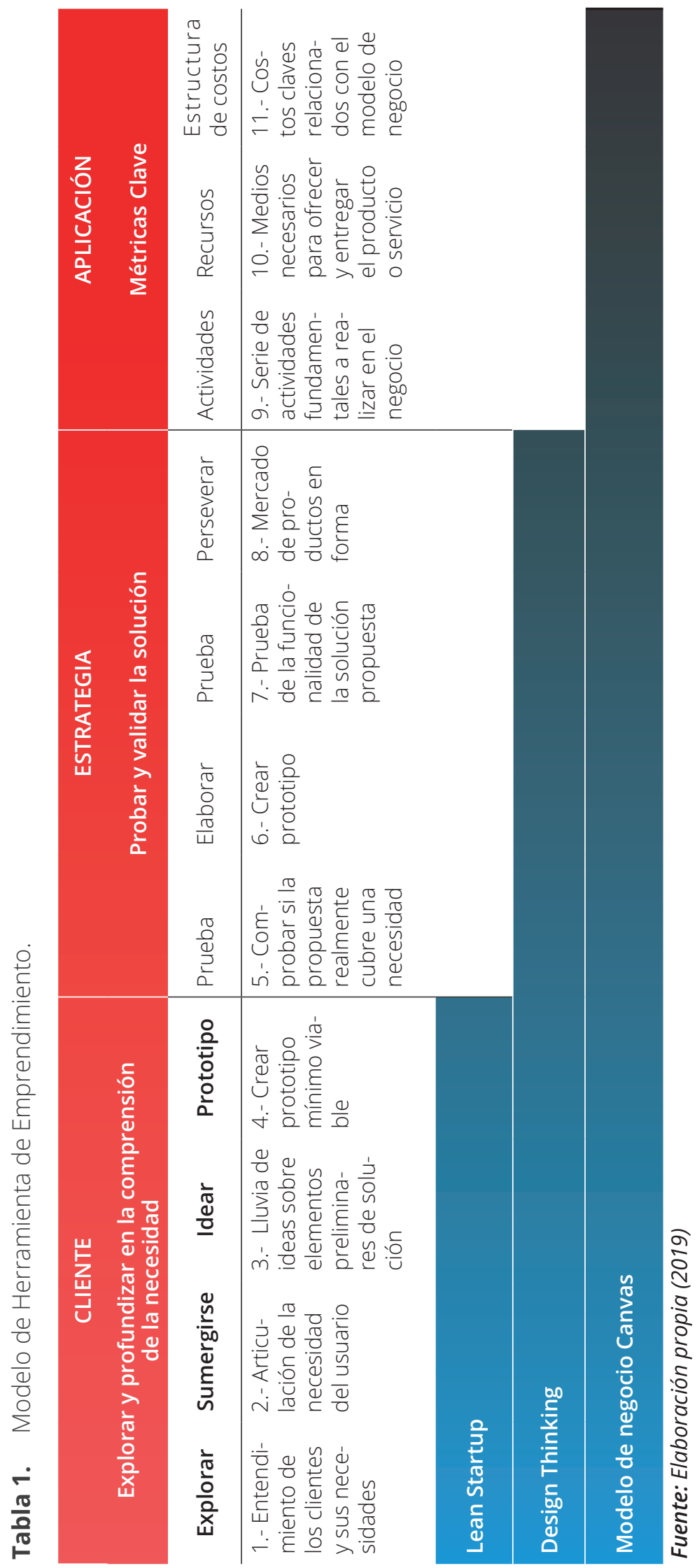




\section{Elementos Tecnológicos que Complementan la Propuesta del Modelo de Herramienta de Emprendimiento}

En los ultimos años los modelos Design Thinking, Lean Startup y Business Model CANVAS han presentado varios cambios debido a las innovaciones digitales al permitir la aplicación de dichos modelos de forma más sencilla y clara, de manera que los emprendedores e inversionistas identifican de manera mas rapida la esencia de propuestas, proyectos y startups. Estas herramientas digitales dada la propuesta de Herramienta de Emprendimiento, son una área de oportunidad para complementarla y eficientarla, se pueden indentificar:

a. Plantilla business model canvas. La plantilla fue elaborada y publicada por Marcelo Pizarro, en ella es posible ir agregando los elementos del modelo de negocios y luego se puede descargar, compartir o exportar en pdf u otros formatos.

b. Canvanizer. Es una plataforma que te permite publicar en una plantilla el modelo de negocio, en ella es posible generar y mover notas, generar variaciones, compartir con el equipo de trabajo y sincronizar a todos los usuarios (Canvanizer, 2018)

c. Strategyzer. Esta plataforma permite analizar modelos de negocio de tal manera que permite diseñar, realizar pruebas, identificar elementos potenciales y evaluar propuestas en tiempo real con el equipo de trabajo; al mismo tiempo, ofrece también una app la cual permite conocer las necesidades de los emprendimientos (Strategyzer AG, 2014).

d. Modelo negocios Business Model CANVAS e SWOT. Es una aplicación que permite crear nuevas plantillas, agregar Post-its e incluso exportar el tablero a correo electrónico o imprimir (Google, 2019).

e. Business Model Fiddle. A través de esta aplicación es posible generar plantillas personalizadas: agregando información, coloreando, filtrando y ordenando artículos de manera rápida y sencilla; permitiendo compartir modelos de negocios de forma controlada (bmfiddle, 2019).

Es importante resaltar que, el solo hecho de llenar los cuadros de la plantilla que se presentan en las diversas herramientas digitales, no asegura el éxito ya que, la calidad y profundidad de la información recopilada sigue siendo el producto básico.

Aunque el modelo de negocio Business Model CANVAS es un referente para emprendedores y grandes compañías, su aplicación bajo un enfoque de Lean Startup representa una oportunidad de desarrollo de emprendimientos -principalmente de base tecnológica- que, complementado con el modelo Design Thinking, permite innovar sobre un modelo de negocio, respondiendo mejor a las necesidades de un startup, independientemente del estado en que ésta se encuentre.

Se considera que el modelo aquí propuesto, puede ser implementado más que por empresas, por aquellas 
personas que buscan una metodología para llevar a cabo la creación de un producto o servicio. Si bien, no se ha realizado una prueba empírica de su aplicación, la validación de las tres herramientas con base en las cuales se fundamenta la propuesta, permite considerarla como viable; no obstante, se considera realizar una segunda investigación que permita demostrar su funcionamiento.

\section{Conclusiones}

En la actualidad el emprendimiento representa un área de oportunidad para todos aquellos que deseen emprender un negocio, transformando ideas en productos de alto valor agregado. Un modelo de negocios es el método visual que permite analizar y optimizar la evolución de un emprendimiento, sin embargo, aunque existen diversos modelos de negocio para desarrollar proyectos sostenibles, en su mayoría estos carecen de métodos para las innovaciones transformadoras y disruptivas, que requieren un proceso iterativo de "investigar y aprender".

No obstante, es posible identificar tres instrumentos revolucionarios para el diseño e innovación de empresas y productos: el modelo Design thinking, Lean startup y Business model canvas; los cuales apoyan al emprendedor a sobreexplotar sus proyectos.

Como proceso de arranque se tiene el modelo Design thinking el cual en su raíz se enfoca en resolver problemas al hacer coincidir las necesidades con lo que es tecnológicamente factible, avanza hacia estos objetivos a través de un enfoque iterativo que involucra la empatía del cliente y el uso de prototipos simples; este enfoque iterativo incorpora muchas de las características del modelo Lean Startup el cual, ofrece una metodología estándar para las innovaciones que requieren una estrategia de aprendizaje, ya que necesitan buscar un modelo de negocio mientras se mantienen las innovaciones ejecutadas en su modelo actual. El modelo de negocio Business Model CANVAS por su parte, implementa un enfoque en cinco áreas: llegar al problema correcto, enfocarse en los clientes correctos, separar los atributos de la solución, imaginar el prototipo mínimo viable y cuestionar los supuestos sobre canales, estructura de costos y tasas de adopción para la nueva innovación.

Como se observa, el modelo Design thinking, Lean startup y Business Model CANVAS podrían convertirse en el proceso de gestión de proyectos estándar para innovaciones transformadoras y disruptivas; al ayudar en la toma de decisiones, la planificación, el estudio sobre la competencia así como, a resolver problemas complejos enfocando al usuario o cliente.

Finalmente, estas herramientas han evolucionado el emprendimiento, lo cual ha permitido el desarrollo de la propuesta del Modelo de Herramienta de Emprendimiento, aquí presentada, misma que como puede observarse, permite aplicar a través de un solo modelo tres métodos, fortaleciendo el proceso previo al emprendimiento.

Lo siguiente a esta investigación es la implementación de la propuesta del 
modelo, la cual se llevará a cabo en el segundo semestre del año dos mil viente, en la materia de Desarrollo de Emprendedores, que se imparte en una escuela de negocios (Facultad de Contaduría y Administración de la Universidad Veracruzana en México) en las carreras de administración y sistemas computacionales administrativos, con lo que se pretende evaluar la pertinencia de dicho modelo.

\section{Referencias}

Amber E. Johnson, Laura Winner, Tanya Simmons, Shaker M. Eid, Robert Hody, Angel Sampedro, Kapil Parakh.(2014).Using blnnovative Methodologies From Technology and Manufacturing Companies to Reduce Heart Failure Readmissions. American Journal of Medical Quality, 31(3), 272-278. https://doi. org/10.1177/1062860614562627

Blank, S., Blank, S. G., \& Dorf, B. (2012). The Startup Owner's Manual: The Step-bystep Guide for Building a Great Company. K\&S Ranch, Incorporated.

Bmfiddle. (2019). Business Model Fiddle. The Free Business Model Canvas Playground. Obtenido de Business Model Fiddle: http://bmfiddle.com

Bosma, Niels; Jones, Kent.; Autio, Erkko y Levie, Jonathan (2008).Global Entrepreneurship Monitor: 2007 Executive Report. Wellesley. Babson College and London Business School. 65p

Brown, T. (2008, junio). Design Thinking, June 2008(R0806E). Recuperado a partir de https://hbr.org/2008/06/ design-thinking

Brown, T. (2009). Change by Design: How Design Thinking Transforms Organizations and Inspires Innovation (First
Edition). Broadway, New York: Harper Collins.

Canvanizer. (2018). HOW TO USE CANVANIZER. Obtenido de Canivanizer: https://canvanizer.com/tutorials

Carlgren, L., I. R., \& Elmquist, M. (2016). Framing Design Thinking: The Concept in Idea and Enactment. Creativity and Innovation Management, 25, 38-57. https://doi.org/10.1111/ caim.12153

Cervilla, M. \& Puente, R.. (2013, abril ) abril). Modelos de negocio de emprendimientos por y para la base de la pirámide. Revista de Ciencias Sociales (RCS), XIX, 289-308.

Craig E. Armstrong. (2016). Running Lean Startup in the Classroom: From Idea to Experiment in 1 Week. Management Teaching Review, 2(2), 132-140. https:// doi.org/10.1177/2379298116683215

Craig E. Armstrong. (2016). Running Lean Startup in the Classroom: From Idea to Experiment in 1 Week. Management Teaching Review, 2(2),132-140. https:// doi.org/10.1177/2379298116683215

Demil, B. \& Lecocq, X. (2009). Evolución de modelos de negocio: Hacia una visión de la estrategia en términos de coherencia dinámica. Universia Business Review, 1, 86-107.

Fino Garzón, D. M. (2013). Innovación en modelos de negocio: metodología Lean Canvas en una Startup de base Tecnológica. Obtenido de e-prints in library \& information science: http:// eprints.rclis.org/22429/1/UMNGAGG48FS-Diego\%20Mauricio\%20Fino\%20 Garzón-Ensayo\%20v.5.pdf

Formichella, M. (2004) El concepto de emprendimiento y su relación con la educación, el empleo y el desarrollo local. INTA. Rivadavia 1439 (1033) Buenos Aires, Argentina 
Google. (2019). play google store. Obtenido de Google Payments: https://play. google.com/store/apps/details?id=com.thirdmobile.modelcanvas

Hisrich, Robert; Peters, Michael y Shepherd,Dean(2006). Entrepreneurship, 7th Edition. New York: McGraw-Hill/ Irwin. 672p.

IESA (2008).Global Entrepreneurship Monitor: informe ejecutivo, Venezuela 2007-2008, Caracas: Instituto de Estudios Superiores de Administración, 73p.

Maurya, A. (2012). Running Lean: Iterate from Plan A to a Plan That Works. O'Reilly Media, Incorporated.

Müller, R., K. T. (2011). Understanding Design Thinking: A Process Model Based on Method Engineering (Vol. 13th, pp. 493-498). Presentado en the 13th International Conference on Engineering and Product Design Education, LONDON, UK: The Design Society a worldwide community. Recuperado a partir de https://www. designsociety.org/publication/30932/ understanding_design_thinking_a_ process_model_based_on_method_ engineering

Osterwalder, A., \& Pigneur, Y. (2011). Generación de modelos de negocios (Primera Edición). Barcelona España: Deusto.

Pérez Paredes, A., Cruz de los Ángeles, J., Guatemala Villalobos, A., \& Juárez Fonseca, V. (2018). Importancia de los pronósticos en la toma de decisiones en las MIPYMES. Revista GEON (Gestión, Organizaciones $Y$ Negocios), 5(1), 97-114. https://doi. org/10.22579/23463910.17

Razzouk R., V. S. (2012).What Is Design Thinking and Why Is It Import- ant?, 82(3), 330-348. https://doi.org/ doi.org/10.3102/0034654312457429

Ries, E., \& Julián, J. S. (2012). El método Lean Startup: Cómo crear empresas de éxito utilizando la innovación continua. Grupo Planeta. Shore, J. (2007). The Art of Agile Development: Pragmatic Guide to Agile Software Development. O'Reilly Media Stanford d.school.(2010). Bootcamp Bootl eg. Recuperado a partir de https://dschool.stanford.edu/resources/the-bootcamp-bootleg

Strategyzer AG. (Diciembre de 2014). Strategyzer. Obtenido de Strategyzer: https://www.strategyzer.com/app

Thoring K., R. M. M. (2012). Design Thiking vs Lean startup: A comparison of two user-driven innovation strategies (pp. 151-161). Presentado en Proceedings of the DMI 2012 International Research Conference: Leading Innovation through Design, BOSTON, MA. USA: Design Management Institute. Recuperado a partir de https:// dmi.site-ym.com/store/ViewProduct. aspx?id=3263535

Torralba Flores, A., Pérez Paredes, A., \& Martínez Ángeles, D. (2017). Fracaso emprendedor, como experiencia de aprendizaje para empresarios del municipio de Puebla. Revista GEON (Gestión, Organizaciones Y Negocios), 4(2), 25-33. https:// doi.org/10.22579/23463910.21

Vianna, Y., M. V., Adler, I. K., Brenda Lucena, \& Beatriz Russo. (2016). Design Thinkin Innovación en los Negocios (First Edition).

Woodilla, J., U. J.-S., \& Çetinkaya, M. (2013). Design Thiking: Past, Present and Possible Futures. Creativity and Innovation Management, 22, 121-146. https://doi.org/10.1111/caim.12023 